H. MUTO

KODAI MATH. J.

18 (1995). 266-274

\title{
SOBOLEV INEQUALITY AND STABILITY OF MINIMAL SUBMANIFOLDS
}

\author{
Hideo Muto
}

\section{Introduction}

Let $M^{n}$ be an $n$ dimensional connected minimal submanifold in an $(n+l)$ dimensional simply connected space form $\bar{M}^{n+l}(b)$ with constant nonpositive sectional curvature $b$. We denote the $L^{p}$ norm of a function $f$ by $\|f\|_{p}$. Sobolev inequalities of the following types play important roles in studying stability of $M$ in $\bar{M}$ :

$$
\|f\|_{n /(n-1)} \leqq A_{1}\|\nabla f\|_{1} \quad \text { for all } f \in C_{0}^{\infty}(M),
$$

in particular, when $n>2$,

$$
\|f\|_{2 n /(n-2)} \leqq A_{2}\|\nabla f\|_{2} \quad \text { for all } f \in C_{0}^{\infty}(M) .
$$

We notice that (i) (1) is also called an isoperimetric inequality, (ii) $A_{2} \leqq$ $(2(n-1) / n-2) A_{1}$, and (iii) when $M^{n}$ is a bounded domain in $\bar{M}^{n}(b), A_{1}=A_{1}(n)$ and $A_{2}=A_{2}(n)$ have the following asymptotic behaviors as $n$ tends to $\infty$ :

$$
A_{1}=A_{1}(n)=\left(\frac{1}{n}\right)^{(n-1) / n} \omega_{n-1}^{-1 / n} \sim \frac{1}{\sqrt{2 \pi e}} \frac{1}{\sqrt{ } n},
$$

and

$$
A_{2}=A_{2}(n)=\frac{2}{\sqrt{n(n-2)}} \omega_{n-1}^{-1 / n} \sim \frac{2}{\sqrt{\pi e}} \frac{1}{\sqrt{n}} .
$$

Here $\omega_{n-1}$ is the volume of $(n-1)$ dimensional unit sphere $S^{n-1}(1)$.

D. Hoffman-J. Spruck [7] derived (2) from (1) with constant $A_{1}=A_{1}(n) \sim$ const. $2^{n} \sqrt{n}$ for minimal submanifolds in $\boldsymbol{R}^{N}$ (see also J. H. Michael - L. M. Simon [11]).

On the other hand, S-Y. Cheng-P. Li-S. T. Yau [3] gave a comparison theorem for the heat kernel of the Laplacian $\Delta$ and P. Li-G. Tian [8] showed a similar comparison theorem for the heat kernel of the Laplacian of an algebraic subvariety in a complex projective space. We point out that E. B. Davies [4] derived a Sobolev inequality of type (2) from can estimate of the heat kernel. But the constant is not given concretely.

Received July 4, 1994. 
In this paper, we give the constant explicitly (Theorem 3) and prove the following :

THEOREM 1. Let $M^{n}$ be an $n$ dimensional minimal submanifold $(n>2)$ in $\bar{M}^{n+l}(b)(b \leqq 0)$. Then we have that

$$
\|f\|_{2 n /(n-2)} \leqq \frac{4 \sqrt{6}}{\pi}\|\nabla f\|_{2} \quad \text { for all } f \in C_{0}^{\infty}(M) .
$$

THEOREM 2. Let $M^{n}$ be an $n$ dimensional algebraic subvariety $(n>1)$ with a singular set $\Sigma_{M}$ in an $(n+l)$ dimensional complex projective space $\boldsymbol{C} P^{n+l}(l>0)$ with sectional curvature $K, 1 \leqq K \leqq 4$. Then for the induced Riemannian metric on $M^{n} \backslash \Sigma_{M}$ from the standard Fubini-Study metric on $C P^{n+l}$, we have

$$
\|f\|_{2 n /(n-1)}^{2} \leqq\left(\frac{8 \sqrt{6}}{\sqrt{\pi}}\right)^{2} e^{1 / 4 n} \max \left\{\frac{\pi^{2}}{4}, n\right\} \omega_{2 n}^{-1 / n}\left(\|\nabla f\|_{2}^{2}+\|f\|_{2}^{2}\right),
$$

for all $f \in C_{0}^{\infty}\left(M \backslash \Sigma_{M}\right)$.

As geometric applications of Theorem 1, we study stability of a minimal submanifold in $\bar{M}^{n+l}(b)$ in section 3 .

\section{Proof of Theorems 1 and 2}

We denote the operator norm of a linear operator $H$ of $L^{p}$ to $L^{q}$ by $\|H\|_{q, p}$. Let $\Omega$ be an $n$ dimensional compact Riemannian manifold. Let $H$ be a positive definite elliptic differential operator on $\Omega$ and $H_{t}=e^{-t H}$ be the semi-group generated by $H$ (under Dirichlet boundary condition if $\Omega$ has boundary) with positive kernel function $H_{\Omega}(t, x, y)$. Then the following inequalities (1) and (2) are equivalent to each other.

(1) $H_{\Omega}(t, x, y) \leqq \alpha t^{-n / 2}$ for all $t>0$ and $x, y \in \Omega$,

(2) $\left\|H_{t}\right\|_{\infty, 1} \leqq \alpha t^{-n / 2}$ for all $t>0$.

When $H$ is a Laplacian $\Delta, H_{\Omega}(t, x, y)$ is the heat kernel $p_{\Omega}(t, x, y)$ of $\Delta$ and $p_{\Omega}$ and $e^{-t \Delta}$ satisfy

$$
\begin{array}{ll}
\int_{\Omega} p_{\Omega}(t, x, y) d y \leqq 1 & \text { for all } t>0 \text { and } x \in \Omega, \\
\left\|e^{-t \Delta}\right\|_{p, p} \leqq 1 & \text { for all } t>0 \text { and } 1 \leqq p \leqq \infty, \\
\left\|\Delta^{1 / 2} f\right\|_{2}=\|d f\|_{2} & \text { for all } f \in C_{0}^{\infty}(\Omega) .
\end{array}
$$

The following theorem is proved in E. B. Davies [4] (Theorem 2.4.2). But the constant is not given concretely.

THEOREM 3. Let $\Omega$ be an $n$ dimensional compact Riemannian manifold with boundary $(n>2)$. Assume that 


$$
\left\|H_{t}\right\|_{p, p} \leqq 1 \quad \text { for all } t>0 \text { and } 1 \leqq p \leqq \infty,
$$

and there exists a positive constant $\alpha$ such that

Then we have

$$
\left\|H_{t}\right\|_{\infty, 1} \leqq \alpha t^{-n / 2} \quad \text { for all } t>0 \text {. }
$$

$$
\|f\|_{2 n /(n-2)} \leqq \frac{8 \sqrt{6}}{\sqrt{\pi}} \alpha^{1 / n}\left\|H^{1 / 2} f\right\|_{2} \quad \text { for all } f \in C_{0}^{\infty}(\Omega) .
$$

Proof. By Riesz interpolation theorem, we have for all $p \in[1, \infty]$ and $t>0$,

$$
\left\|H_{t}\right\|_{\infty, p} \leqq \alpha^{1 / p} t^{-n / 2 p} .
$$

In particular, for $p \in[1, n)$ and $f \in L^{p}$, we can define $L$ by

$$
L f=\Gamma(1 / 2)^{-1} \int_{0}^{\infty} t^{-1 / 2} H_{t} f d t
$$

where $\Gamma(x)$ is the Gamma function and $\Gamma(1 / 2)=\sqrt{\pi}$. We notice here that $L_{\mid C_{0}^{\infty}(\Omega)}=\left(H_{1 C_{0}^{\infty}(\Omega)}\right)^{-1 / 2}$. For $f \in L^{p}(1 \leqq p<n)$ and a positive constant $T$, we write $L f$ by

where

$$
L f=g_{T}+h_{T}
$$

$$
g_{T}=\frac{1}{\sqrt{\pi}} \int_{0}^{T} t^{-1 / 2} H_{t} f d t, \quad h_{T}=\frac{1}{\sqrt{\pi}} \int_{T}^{\infty} t^{-1 / 2} H_{t} f d t .
$$

Define $q(p), C_{1}(p)$ and $M(p)$ by $1 / q(p)=1 / p-1 / n$,

$$
C_{1}(p)=\frac{1}{\sqrt{\pi}} \alpha^{1 / p} \frac{2 p}{n-p} \text { and } M(p)=\frac{4}{\sqrt{\pi}} \alpha^{1 / n}\left(\frac{p}{n-p}\right)^{p / n} .
$$

And define $T_{\lambda}$ for all $\lambda>0$ by $\lambda / 2=C_{1}(p)\|f\|_{p} T_{\lambda}^{(1 / 2)-(n / 2 p)}$. Then we see that $\left\|h_{T_{\lambda}}\right\|_{\infty} \leqq \lambda / 2$ and $\{x \in \Omega:|L f(x)| \geqq \lambda\} \subset\left\{x \in \Omega:\left|g_{T_{\lambda}}(x)\right| \geqq \lambda / 2\right\}$. So we have

$$
\begin{aligned}
\operatorname{vol}\{x \in \Omega:|L f(x)| \geqq \lambda\} & \leqq \operatorname{vol}\left\{x \in \Omega:\left|g_{T_{\lambda}}(x)\right| \geqq \lambda / 2\right\} \\
& \leqq(\lambda / 2)^{-p} \int_{\left.\left|g_{T_{\lambda} \mid \geq \lambda / 2}\right| g_{T_{\lambda}}(x)\right|^{p} d x} \\
& \leqq(\lambda / 2)^{-p}\left\|g_{T_{\lambda}}\right\|_{p}^{p} .
\end{aligned}
$$

On the other hand,

$$
\begin{aligned}
\left\|g_{T_{\lambda}}\right\|_{p} & =\left\|\frac{1}{\sqrt{\pi}} \int_{0}^{T \lambda} t^{-1 / 2} H_{t} f d t\right\|_{p} \\
& \leqq \frac{1}{\sqrt{\pi}} \int_{0}^{T \lambda} t^{-1 / 2}\left\|H_{t} f\right\|_{p} d t \\
& \leqq \frac{2}{\sqrt{\pi}}\|f\|_{p} T_{\lambda}^{1 / 2} .
\end{aligned}
$$


Therefore

$$
\operatorname{vol}\{x \in \Omega:|L f(x)| \geqq \lambda\} \leqq\left(\frac{M(\lambda)}{\lambda}\|f\|_{p}\right)^{q(p)} .
$$

For all $p_{1}$ and $p_{2}\left(1 \leqq p_{1}<2<p_{2}<n\right)$, define $q_{1}, q_{2}$ and $\theta\left(p_{1}, p_{2}\right)$ by $q_{1}=q\left(p_{1}\right), q_{2}=$ $q\left(p_{2}\right)$ and $1 / 2=\left(1-\theta\left(p_{1}, p_{2}\right)\right) / p_{1}+\theta\left(p_{1}, p_{2}\right) / p_{2}$. We here apply Marcinkiewicz interpolation theorem to $L$ for $p_{1}$ and $p_{2}$ above. We use the constant $K$ in $\mathrm{A}$. Zygmund [16] (see also D. Gilbarg-N.S. Trudinger [6], p. 228 and p. 254). Then we have for all $f \in L^{2}$,

Here

$$
\|L f\|_{2 n /(n-2)} \leqq K\left(p_{1}, p_{2}\right) M\left(p_{1}\right)^{1-\theta\left(p_{1}, p_{2}\right)} M\left(p_{2}\right)^{\theta\left(p_{1}, p_{2}\right)}\|f\|_{2} .
$$

$$
K\left(p_{1}, p_{2}\right)=2 q(2)^{1 / q(2)}\left(\frac{\left(p_{1} / 2\right)^{q_{1} / p_{1}}}{\left|q_{1}-q(2)\right|}+\frac{\left(p_{2} / 2\right)^{q_{2} / p_{2}}}{\left|q_{2}-q(2)\right|}\right)^{1 / q(2)} .
$$

Set $F\left(p_{1}, p_{2}, x\right)$ by

$$
\begin{aligned}
F\left(p_{1}, p_{2}, x\right)= & \frac{8}{\sqrt{\pi}}\left(\frac{p_{1}}{2-p_{1}}\left(1-p_{1} x\right)\left(p_{1} / 2\right)^{p_{1} x /\left(1-p_{1} x\right)}\right. \\
& \left.\quad+\frac{p_{2}}{p_{2}-2}\left(1-p_{2} x\right)\left(p_{2} / 2\right)^{p_{2} x /\left(1-p_{2} x\right)}\right)^{1 / 2-x} \\
& \times\left(\frac{p_{1} x}{1-p_{1} x}\right)^{p_{1} x\left(1 / 2-1 / p_{2}\right) /\left(1 / p_{1}-1 / p_{2}\right)} \\
& \times\left(\frac{p_{2} x}{1-p_{2} x}\right)^{p_{2} x\left(1 / p_{1}-1 / 2\right) /\left(1 / p_{1}-1 / p_{2}\right)}
\end{aligned}
$$

Since $\|L f\|_{2 n /(n-2)} \leqq F\left(p_{1}, p_{2}, 1 / n\right)\|f\|_{2}$, we may show

$$
\inf _{1 \leqq p_{1}<2<p_{2}<1 / x} F\left(p_{1}, p_{2}, x\right)<\frac{8 \sqrt{6}}{\sqrt{\pi}} \quad \text { for } x=1 / 3,1 / 4,1 / 5, \cdots .
$$

Since $F(1.7,2.2,1 / 3)=10.3946 \cdots<8 \sqrt{6} / \sqrt{\pi}$, we consider $F(1,5 / 2, x)$ for all $0<x \leqq 1 / 4$. We easily see that

$$
\begin{array}{ll}
F(1,5 / 2,1 / 4)=7.59103 \cdots, & \text { for } 0<x<1, \\
\left(\frac{1}{2}\right)^{x /(1-x)} \leqq 1 & \text { for } 0<x \leqq \frac{1}{4}<\frac{2}{5} \log \frac{4 e}{5}, \\
1<5\left(1-\frac{5}{2} x\right)\left(\frac{5}{4}\right)^{5 x /(2-5 x)} \leqq 5 & \text { for } 0<x \leqq 1 / 2 .
\end{array}
$$

Therefore we have 


$$
F(1,5 / 2, x)<\frac{8 \sqrt{6}}{\sqrt{\pi}} \quad \text { for } x=1 / 5,1 / 6, \cdots
$$

and

$$
\|\phi\|_{2 n /(n-2)} \leqq \frac{8 \sqrt{6}}{\sqrt{\pi}} \alpha^{1 / n}\left\|H^{1 / 2} \phi\right\|_{2} \quad \text { for all } \phi \in C_{0}^{\infty}(\Omega)
$$

To prove Theorems 1 and 2, we prepare some comparison theorems for the heat kernel of the Laplacian. We notice here that the heat kernel $p(t, x, y)$ of Laplacian on a symmetric space depends only on $t>0$ and the distance $d(x, y)$ of $x$ and $y$. So we can write $p(t, x, y)$ on a symmetric space as $p(t, d(x, y))$.

Theorem 4 (S-Y. Cheng-P. Li-S. T. Yau [3]). Let $M^{n}$ be an $n$ dimensional minimal submanifold of $\bar{M}^{n+l}(b)(l>0, b \leqq 0)$ and $\Omega$ compact domain in $M$ and any $p \in \Omega$. Let $p(t, x, y)$ be the heat kernel of the Laplacian on $M$ under Dirichlet boundary condition. We define the extrinsic outer radius at $p$ by

$$
a=\sup _{z \in \Omega} d(p, z)
$$

Then

$$
p(t, p, y) \leqq \bar{p}_{a}(t, \ddot{d}(p, y))
$$

for all $y \in \Omega$ and $t \in(0, \infty)$. Here $\bar{d}(p, z)$ is the distance function on $\bar{M}$ and $\bar{p}_{a}(t, d(p, y))$ stands for the heat kernel under Dirichlet boundary condition on the ball centered at some fix point with radius $a$ in $\bar{M}^{n}(b)$.

Theorem 5 (P. Li-G. Tian [8]). Let $M^{n}$ be an $n$ dimensional embedded algebraic submanifold of $C P^{n+l}$. Let $p(t, x, y)$ be the heat kernel of $M$ with respect to the induced metric. When $M$ has boundary, $p(t, x, y)$ is taken to be the heat kernel under Dirichlet boundary condition. Then for all $x, y \in M$ and $t \in(0, \infty)$, we have

$$
p(t, x, y) \leqq \bar{p}(t, \bar{d}(x, y)) .
$$

Here $\bar{p}(t, \bar{d}(x, y))$ is the heat kernel of $C P^{n}$ and $\bar{d}(x, y)$ is the distance function of $\bar{M}$.

Proof of Theorem 1. Let $p_{\Omega}(t, x, y)$ be the heat kernel of the Laplacian of a bounded domain $\Omega$ in $M$ under Dirichlet boundary condition. By Theorem 4 , we have

$$
p_{\Omega}(t, x, y) \leqq \frac{1}{(4 \pi t)^{n / 2}} \quad \text { for all } x, y \in \Omega \text { and } t>0 .
$$

So by Theorem 3 , we have 


$$
\|f\|_{2 n /(n-2)} \leqq \frac{4 \sqrt{6}}{\sqrt{\pi}}\|\nabla f\|_{2} \quad \text { for all } f \in C_{0}^{\infty}(M),
$$

Proof of Theorem 2. Let $Q$ be a bounded domain $M \backslash \Sigma$ and let $\bar{p}(t, \bar{d}(\bar{x}, \bar{y}))$ be the heat kernel of the Laplacian on $\boldsymbol{C} P^{n}$ with the Fubini-Study metric. The kernel function $H(t, x, y)$ of $e^{-t H}$ for $H=(-\Delta+1)$ on $\Omega$ under Dirichlet boundary condition is $e^{-t} p_{\Omega}(t, x, y)$. By Theorem 5 , we have

$$
p_{\Omega}(t, x, y) \leqq \bar{p}(t, 0) \quad \text { for all } x, y \in \Omega \text { and } t>0 .
$$

On the other hand, a heat kernel $\tilde{p}(t, \tilde{x}, \tilde{y})$ on an $m$ dimensional compact connected Riemannian manifold $\left(\tilde{M}^{m}, \tilde{g}\right)$ with nonnegative Ricci curvature satisfies

$$
\tilde{p}(t, \tilde{x}, \tilde{x}) \leqq \tilde{p}(t+s, \tilde{x}, \tilde{y})\left(\frac{t+s}{t}\right)^{m / 2} \exp \left(\frac{\tilde{d}^{2}(\tilde{x}, \tilde{y})}{4 s}\right),
$$

for all $t, s>0$ and $\tilde{x}, \tilde{y} \in \tilde{M}$ (see P. Li and S. T. Yau [10]). Let $\tilde{D}$ be the diameter of $\tilde{M}$. Integrating the both sides in $\tilde{y} \in \tilde{M}$ and substituting $s=\widetilde{D}^{2}$, we have

Therefore we have

$$
\begin{aligned}
e^{-t} \tilde{p}(t, \tilde{x}, \tilde{x}) & \leqq e^{-t}\left(\frac{t+\tilde{D}^{2}}{t}\right)^{m / 2} e^{1 / 4} \frac{1}{\operatorname{vol}(\tilde{M}, \tilde{g})} \\
& \leqq\left(e^{1 / 2 m} \max \left\{\tilde{D}^{2}, \frac{m}{2}\right\}\right)^{m / 2} \frac{1}{\operatorname{vol}(\tilde{M}, \tilde{g})} t^{-m / 2} .
\end{aligned}
$$

$$
\begin{aligned}
H(t, x, y) & \leqq e^{-t} \bar{p}(t, 0) \\
& \leqq\left(e^{1 / 4 n} \max \left\{\frac{\pi^{2}}{4}, n\right\}\right)^{n} \frac{1}{\operatorname{vol}\left(\boldsymbol{C} P^{n}\right)} t^{-n} \\
& =\left(e^{1 / 4 n} \max \left\{\frac{\pi^{2}}{4}, n\right\}\right)^{n} \frac{1}{2 \pi \omega_{2 n}} t^{-n}
\end{aligned}
$$

Applying Theorem 3 to $H$, we have for all $f \in C_{0}^{\infty}\left(M \backslash \Sigma_{M}\right)$,

$$
\|f\|_{2 n /(n-1)}^{2} \leqq\left(\frac{8 \sqrt{6}}{\sqrt{\pi}}\right)^{2} e^{(1 / 4 n)} \max \left\{\frac{\pi^{2}}{4}, n\right\} \omega_{2 n}^{-1 / n}\left(\|\nabla f\|_{2}^{2}+\|f\|_{2}^{2}\right) .
$$

\section{Applications}

A compact minimal submanifold $\Omega$ with boundary is a critical point of volume functional for variations fixing boundary. Define the index of $\Omega$, index $(\Omega)$, by the number of negative eigenvalues of the elliptic differential operator $J$ called a Jacobi operator arising from the second variation of the 
volume functional. For a noncompact minimal submanifold $M$, define the index of $M$, index $(M)$, by $\lim _{\imath \rightarrow \infty}\left\{\right.$ index $\left(\Omega_{\imath}\right):\left\{\Omega_{i}\right\}$ is an exhaustion of $\left.M\right\}$. And $M$ (resp. $\Omega$ ) is said to be stable when $\operatorname{index}(M)=0(\operatorname{resp}$. index $(\Omega)=0)$.

As geometric applications of Theorem 1, we give an estimate of the index of a minimal submanifold $M^{n}$ in $\bar{M}^{n+l}(b)(b \leqq 0)$ and give some conditions for $M^{n}$ in $\boldsymbol{R}^{n+l}$ to be an $n$ dimensional plane.

For index $(M)$, J. Tysk [15] showed the following theorem using Sobolev inequality of type (2) derived from Sobolev inequality of type (1).

We denote the second fundamental form of $M$ in $\bar{M}$ by $B$.

Theorem 6 (J. Tysk [16]). Let $M^{n}$ be an $n$ dimensional oriented complete minimal hypersurface in $\boldsymbol{R}^{n+1}(n>2)$. Then we have

$$
\operatorname{index}(M) \leqq \frac{n}{\omega_{n-1}}\left(\frac{\sqrt{e}(n-1) 2^{2 n+3}}{n-2}\right)^{n} \int_{M}|B|^{n} .
$$

So we can prove the following theorem in the same way as in [13]. Since we only replace the Sobolev inequality of type (2) in [13] by the inequality in Theorem 1, we omit the proof. Set $f_{+}=\max (f, 0)$.

THEOREM 7. Let $M^{n}$ be an $n$ dimensional oriented noncompact complete minimal hypersurface $(n>2)$ in $\bar{M}^{n+1}(b)(b \leqq 0)$. Then we have

$$
\text { index }(M) \leqq e^{n / 2}\left(\frac{4 \sqrt{6}}{\pi}\right)^{n} \int_{M}\left(|B|^{2}+n b\right)_{+}^{n / 2} .
$$

We next study the stability of a minimal submanifold in $\boldsymbol{R}^{n+l}$.

THEOREM 8 (P.H. Bérard [1]). Let $M^{n}$ be an $n$ dimensional noncompact complete minimal submanifold $(n>2)$ in $\boldsymbol{R}^{N}$. Set

$$
\begin{aligned}
\alpha(n, N) & =\frac{2}{(n+2)(N-n)-2}, \quad \beta(n, N)=2-\frac{1}{N-n}, \\
C_{1}(n) & =2^{n-1} \pi(n+1)^{(n+1) / n} \omega_{n}^{-1 / n} /(n-1), \\
C_{2}(n) & =2 C_{1}(n) \frac{n-1}{n-2}, \\
C_{3}(n, N) & =2 \frac{n+\alpha(n, N)-1}{n^{2} C_{2}(n)^{2} \beta(n, N)} .
\end{aligned}
$$

If $\|B\|_{n}^{2}<C_{3}(n, N)$, then $M$ is an $n$ dimensional plane.

In [1], P.H. Bérard used an inequality of J. Simons [13] and a Sobolev inequality of type (2) in section 1. Replacing an inequality of J. Simons by $\mathrm{T}$. Okayasu [12] and a Sobolev inequality of type (2) by Theorem 1, we can improve the above condition. 
THEOREM 9. Let $M^{n}$ be an $n$ dimensional noncompact complete minimal submanifold $(n>2)$ in $\boldsymbol{R}^{N}$. If

$$
\|B\|_{n}^{2}<4 \frac{n+(2 / n)-1}{n^{2} \beta(n, N)}\left(\frac{\pi}{4 \sqrt{ } 6}\right)^{2},
$$

then $M$ is an $n$ dimensional plane.

Under a weaker condition, we show that $M$ is stable as follows (see J. Spruck [14]).

THEOREM 10. Let $M^{n}$ be an $n$ dimensional noncompact complete minimal submanifold $(n>2)$ in $\bar{M}^{n+l}(b)(b \leqq 0)$. If

$$
\left\|\sqrt{\left(|B|^{2}+n b\right)_{+}}\right\|_{n} \leqq \frac{\pi}{4 \sqrt{6}},
$$

then $M$ is stable.

In particular, if $M^{n}$ is a minimal hypersurface in $\boldsymbol{R}^{n+1}$ satisfying $\|B\|_{n} \leqq$ $\pi / 4 \sqrt{ } 6$ and $\|B\|_{2}<\infty$, then $M$ is an $n$ dimensional hyperplane.

Proof. Let $\Omega$ be a bounded domain in $M$. By Theorem 1, for a variation vector field $X$ on $\Omega$ in $M$ fixing boundary, $u=|X|$ satisfies that $u_{\mid \partial \Omega}=0$ and

$$
\begin{aligned}
V^{\prime \prime}(0)= & \int_{\Omega}(J X, X) \\
\geqq & \int_{\Omega}|\nabla u|^{2}-\left(|B|^{2}+n b\right)+u^{2} \\
\geqq & \left(\frac{\pi}{4 \sqrt{6}}\right)^{2}\left(\int_{\Omega} u^{2 n /(n-2)}\right)^{(n-2) / n} \\
& -\left(\int_{\Omega}\left(|B|^{2}+n b\right)_{+}^{n / 2}\right)^{2 / n}\left(\int_{\Omega} u^{2 n /(n-2)}\right)^{(n-2) / n} \\
\geqq & \left(\left(\frac{\pi}{4 \sqrt{6}}\right)^{2}-\left\|\sqrt{\left.\left(|B|^{2}+n b\right)_{+}\right)}\right\|_{n}^{2}\right)\left(\int_{\Omega} u^{2 n /(n-2)}\right)^{(n-2) / n} \\
\geqq &
\end{aligned}
$$

Therefore, every bounded domain in $M$ is stable. And a complete stable minimal hypersurface in $\boldsymbol{R}^{n+1}$ with $\|B\|_{2}<\infty$, it is a hyperplane (see M. do Carmo and C. K. Peng [5]). 


\title{
REFERENCES
}

[1] P.H. BÉRARd, Remarques sur l'equations de J. Simons, Differential Geometry, Longman Sci. \& Tech., Harlow, 1991, 47-57.

[2] P.H. Bérard and G. Besson, On the number of bound states and estimates on some geometric invariants, Lect. Notes in Math., 1324, Springer-Verlag, BerlinHeidelberg-New York-Tokyo, 1988.

[3] S.-Y. Cheng, P. Li ANd S.-T. YAU, Heat equations on minimal submanifolds and their applications, Amer. J. Math., 106 (1984), 1033-1065.

[4] E. B. Davies, Heat kernels and spectral theory, Cambridge Univ. Press, 1989.

[5] M. Do Carmo and C.K. Peng, Stable complete minimal hypersurfaces, Proceedings of the 1980 Beijin Symposium on Differential Geometry and Differential Equations, ed. by S.S. Chern and W.W. Tsün, Science Press, Beijing; Gordon and Breach Science Publishers, New York, 1982, 1349-1358.

[6] D. Gilbarg and N.S. Trudinger, Elliptic Partial Differential Equations of Second Order, Springer-Verlag Berlin-Heidelberg-New York-Tokyo, 1983.

[7] D. Hoffman ANd J. SpRuck, Sobolev and isoperımetric inequalities for Riemannian submanifolds, Comm. Pure Appl. Math., 27 (1974), 715-727.

[8] P. Li AND G. TIAN, On the heat kernel of the Bergman metric of algebraic varieties, preprint.

[9] P. LI AND S.-T. YAU, On the Schrödinger equation and the eigenvalue problem, Comm. Math. Phys., 88 (1983), 309-318.

[10] P. Li AND S.-T. YAU, On the parabolic kernel of the Schrödinger operator, Acta Math., 156 (1986), 153-201.

[11] J.H. Michael And L. M. Simon, Sobolev and mean-value inequalities on generalized submanifolds of $\boldsymbol{R}^{n}$, Comm. Pure Appl. Math., 26 (1973), 361-379.

[12] T. OKAYASU, Isolation theorem for harmonic maps, preprint.

[13] J. Simons, Minimal varieties in Riemannian manifolds, Ann. of Math., 88 (1968), 62-105.

[14] J. SpRUCK, Remarks on the stability of minımal submanifolds of $\boldsymbol{R}^{n}$, Math. $Z$., 144 (1975), 169-174.

[15] J. Tysk, Finiteness of index and total scalar curvature for minimal hypersurfaces, Proc. Amer. Math. Soc., 105 (1989), 429-435.

[16] A. Zygmund, Trigonometric Series, II, second edition, Cambridge Univ. Psess, 1959.

\author{
Department of Mathematics \\ Faculty of Liberal Arts and Education \\ YAMANASHI UNIVERSITY \\ TAKEDA 4-4-37, KOFU CiTy 400, JAPAN \\ e-mail: hmuto@grape.kkb.yamanashi.ac.jp
}

